\title{
Fetal development of the carotid canal with special reference to a contribution of the sphenoid bone and pharyngotympanic tube
}

\author{
Yohei Honkura ${ }^{1}$, Masahito Yamamoto $^{2}$, José Francisco Rodríguez-Vázquez ${ }^{3}$, Gen Murakam4, \\ Hiroshi Abe ${ }^{5}$, Shin-ichi Abe ${ }^{2}$, Yukio Katori ${ }^{1}$ \\ ${ }^{1}$ Department of Otolaryngology-Head and Neck Surgery, Tohoku University School of Medicine, Sendai, ${ }^{2}$ Department of Anatomy, Tokyo Dental \\ College, Tokyo, Japan, ${ }^{3}$ Department of Anatomy and Embryology, School of Medicine, Complutense University of Madrid, Madrid, Spain, ${ }^{4}$ Division of \\ Internal Medicine, Cupid Clinic, Iwamizawa, ${ }^{5}$ Akita University School of Medicine, Akita, Japan
}

\begin{abstract}
The bony carotid canal is a tube-like bone with a rough surface in contrast to smooth surfaces of the other parts of the temporal bone petrosal portion (petrosa): it takes an impression of the additional, out-sourcing product. No study had been conducted to evaluate a contribution of the adjacent sphenoid and pharyngotympanic tube (PTT) to the carotid canal. We examined sagittal and horizontal histological sections of hemi-heads from 37 human fetuses at 10 to 37 weeks. At 10 to 18 weeks, the future carotid canal was identified as a wide loose space between the cartilaginous cochlea and the ossified or cartilaginous sphenoid elements (ala temporalis and pterygoid). A linear mesenchymal condensation extending between the cochlear wall and ala temporalis suggested the future antero-inferior margin of the carotid canal. This delineation was more clearly identified in later stages. After 25 weeks, 1) the growing pterygoid pushed the PTT upward and, in turn, the PTT pushed the internal carotid artery (ICA) upward toward the petrosa: 2) a membranous ossification occurs in the dense mesenchymal tissue, the latter of which took an appearance of an anterior process of the petrosa; 3 ) the bony process of the petrosa involved the ICA inside or posteriorly. The bony carotid canal was made with membranous ossification in the dense mesenchymal tissue between the petrosa and sphenoid. The mother tissue was detached from the sphenoid by the PTT. The ossification of the septum between the ICA and tympanic cavity seemed to continue after birth.
\end{abstract}

Key words: Carotid canal, Membranous ossification, Sphenoid bone, Ala temporalis, Pterygoid

Received November 27, 2020; Revised January 27, 2021; Accepted March 8, 2021

\section{Introduction}

The bony carotid canal in adults is a tube-like bone with a rough surface in contrast to smooth surfaces of the other parts of the temporal bone petrosal portion (below, abbreviated by "petrosa"): the morphology takes an impression of

\section{Corresponding author:}

Masahito Yamamoto (iD

Department of Anatomy, Tokyo Dental College, Tokyo 101-0061, Japan E-mail:yamamotomasahito@tdc.ac.jp the additional, out-sourcing product after a complete development of the inner ear in the petrosa (Fig. 1). The slowlydeveloped tympanic bone also has a rough external surface. Without the carotid canal and tympanic bone, the anteroinferior aspect of the petrosa would simply reflect a slight convex of the cochlear wall. Our group incidentally demonstrated photos of the bony canal developing much later than any surface structures of the petrosa $[1,2]$ although it was out of their focus.

Usually, the skull base skeleton is simply divided into two parts: bony parts in which endochondral ossification occurs and the other parts with membranous ossification $[3,4]$.

\section{Copyright () 2021. Anatomy \& Cell Biology}

This is an Open Access article distributed under the terms of the Creative Commons Attribution Non-Commercial License (http://creativecommons.org/licenses/by-nc/4.0/) which permits unrestricted non-commercial use, distribution, and reproduction in any medium, provided the original work is properly cited. 


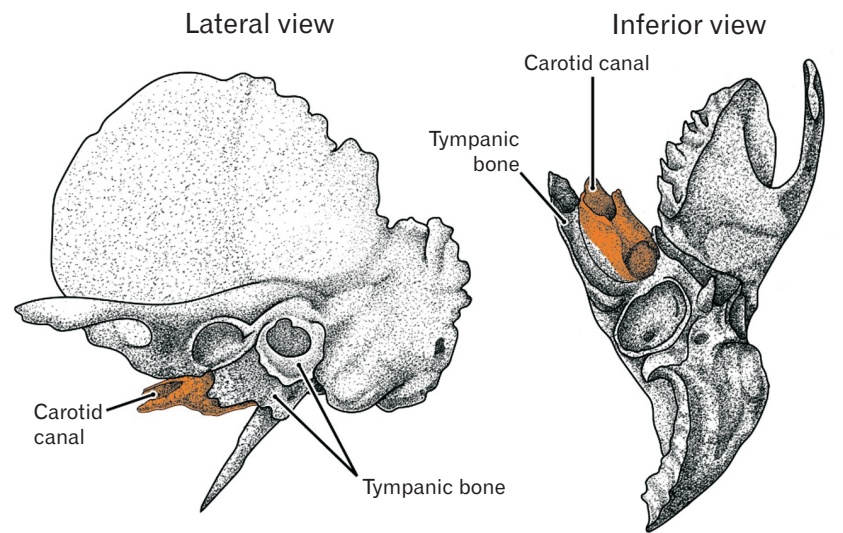

Fig. 1. Temporal bone in adults: the lateral and inferior views. The carotid canal (colored orange) as well as the tympanic bone has rough external surfaces and they appear to additionally attach to the inferolateral aspect of the temporal bone.

However, in the skull base, as typically seen in the pterygoid of the sphenoid, membranous ossification simultaneously occurs at a site adjacent to endochondral ossification [5]. Also in the ear, Michaels et al. (2010) [6] described membranous ossification occurs to provide a lamellar bone along the endolymphatic duct after ossification of the otic capsule cartilage. Because endochondral ossification of the ear requires its cartilage model before midterm and occurs until 21-24 weeks [7-10], the carotid canal is most likely to be established by membranous ossification as in the facial nerve canal [11]. A delayed ossification is known well in the facial nerve canal because of the so-called dehiscence (reviewed by Spector and Ge [12], 1993).

Although the bony carotid canal may be simply believed as a part of the petrosa, no study had been conducted to evaluate a contribution of the adjacent sphenoid and pharyngotympanic tube in the fetal development and growth. In the anterior side of the ear, three elements of the sphenoid are located: 1) the ala temporalis and 2) the alar process, both of which take endochondral ossification and, 3) the pterygoid composed of multiple parts each of which takes endochondral or membranous ossification $[5,13,14]$. Is it likely for the sphenoid elements to contribute the closure of the carotid canal? Consequently, the aim of this study was to examine when and how the internal carotid artery (ICA) is involved into the petrosa without involvement into the sphenoid elements.

\section{Materials and Methods}

This study was conducted in accordance with the Declaration of Helsinki 1995 (as revised in 2013). We examined paraffin-embedded histological sections from 32 fetuses (approximately 12 to 37 weeks of gestational age; 28 to $310 \mathrm{~mm}$ of crown-rump length (CRL). The specimens were categorized into two groups according to age and size: 1) 20 midterm fetuses at 10 to 18 weeks of gestational age (CRL, 19-120 mm) and 2) 17 late-stage fetuses at 25 to 37 weeks (CRL, 200-310 mm).

Serial histological sections of 20 midterm fetuses were part of the large collection kept at the Department of Anatomy of the Complutense University of Madrid, Madrid, and were the results of miscarriages and ectopic pregnancies from the Department of Obstetrics of the University. They had been prepared serially (sagittal, 15 fetuses; horizontal, five fetuses) and stained with hematoxylin and eosin (H\&E), azan or orange $\mathrm{G}$. This study was approved by the Ethics Committee of Complutense University of Madrid (B08/374). Photographs were taken with a Nikon Eclipse 80 (Nikon, Tokyo, Japan).

From 17 late-stage fetuses, we prepared paraffin-embedded sections at 50 or 100 micron interval. The sectional planes were sagittal (12 fetuses) and horizontal (5 fetuses) and, all sections were stained with H\&E. These specimens were parts of the collection of the Department of Anatomy, Akita University, Akita, Japan. These specimens had been donated by their families to the Department from 1975-1985 and preserved in $10 \%(\mathrm{w} / \mathrm{w})$ neutral formalin solution for more than 30 years. The available data were limited to the date of donation and gestational age, but there was no information on family name, the name of the obstetrician or hospital and the reason for abortion. The use of these specimens for research was approved by the ethics committee of Akita University (No. 1428). Photographs were taken with a Nikon Eclipse 80.

Most photographs were taken with a Nikon Eclipse 80, whereas photographs at ultra-low magnification (objective lens less than $\times 1$ ) were obtained using a high-grade flat scanner with translucent illumination (Epson scanner GTX970; Epson, Nagano, Japan).

\section{Results}

\section{Observations of the midterm fetuses}

Sagittal sections from 15 midterm fetuses (12-18 weeks) 

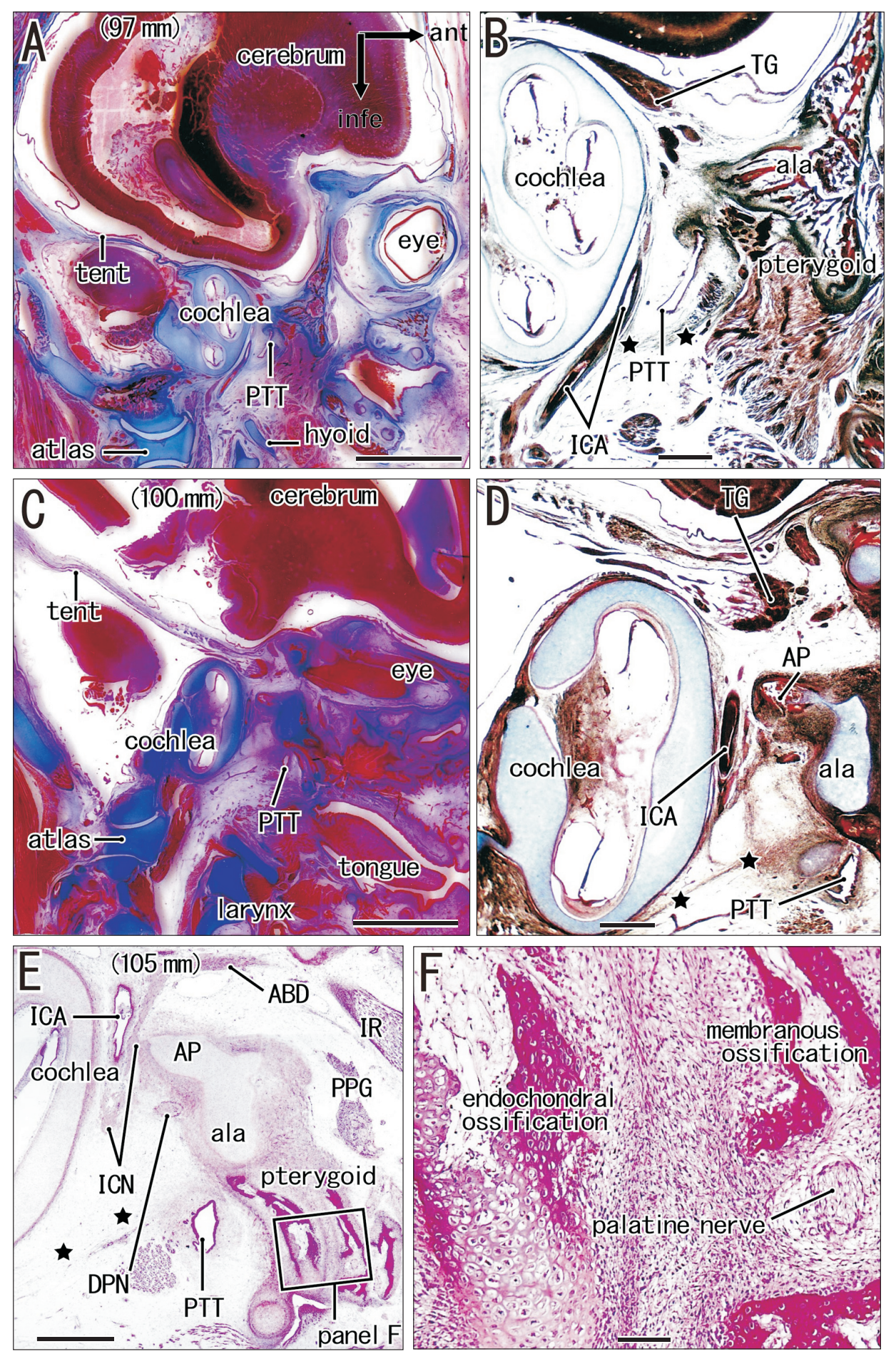

Fig. 2. Initial carotid canal in sagittal sections: a wide gap or loose space between the cochlear wall and the sphenoid. Azan staining (A-D) or H\&E staining (E, F). (A, B) A fetus of crown-rump length (CRL) 97 mm (approximately 13 weeks); (C, D) CRL $100 \mathrm{~mm}$ (13 weeks); (E, F) CRL $105 \mathrm{~mm}$ (14 weeks). (B) and (D) are higher magnification view of the center of (A) and (C), respectively. (F) is a higher magnification view of a square in (E). The left-hand side of each panel corresponds to the anterior side of the head. The ala temporalis of the sphenoid (ala) is ossified in (B) and (D), while it is cartilaginous in (E). In (D), an inferior part of the ala (ala with asterisk) looks like a pterygoid. In contrast, most parts of the pterygoid of the sphenoid is bony (B, E). (F) Shows the pterygoid containing two modes of the ossification. (B, D, E) Stars indicate a linear mesenchymal condensation corresponding to the future antero-inferior margin of the petrosal part of the temporal bone. Scale bars: (A, C) 5 mm; (B, D, E) $1 \mathrm{~mm}$; (F) $0.1 \mathrm{~mm}$. ABD, abducant nerve; AP, alar process; DPN, deep petrosal nerve; ICA, internal carotid artery; IR, inferior rectus of the eye; PPG, pterygopalatine ganglion; PTT, pharyngotympanic tube; tent, tentorium cerebelli; TG, trigeminal ganglion. 
always demonstrated a longitudinal course of the ICA along a slight curvature or convex provided by the cartilaginous anterior cochlear wall (Fig. 2). The ICA ran superiorly along the vertebral column and, near the inferior end of the inner ear cartilage, the ICA turned anteriorly. Thus, the anterior turn of the ICA at the skull base was established until mid-
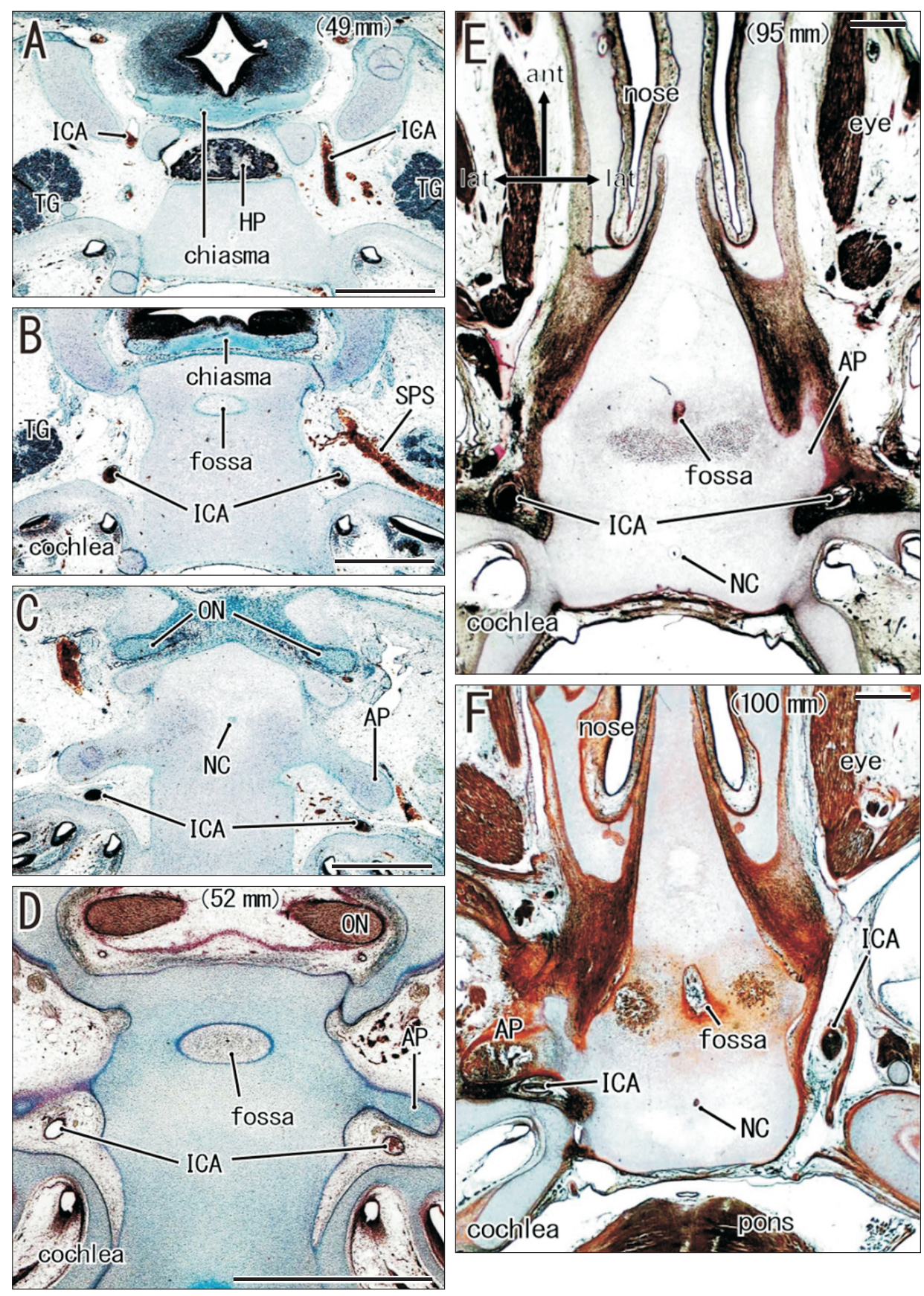

Fig. 3. Initial carotid canal in horizontal sections: a slit surrounded by the cochlear wall, alar process (AP) and basisphenoid. Azan staining. (AC) A fetus of crown-rump length (CRL) $49 \mathrm{~mm}$ (approximately 10 weeks); (D) CRL $52 \mathrm{~mm}$ (10 weeks); (E) CRL $95 \mathrm{~mm}$ (13 weeks); (F) CRL $100 \mathrm{~mm}$ (14 weeks). The upper side of each panel corresponds to the anterior side of the head. The basisphenoid, a large cartilage mass, contains both the notochord (NC) and hypophysial fossa (fossa). The internal carotid artery is surrounded by the petrosa or cochlear wall posteriorly, the AP anteriorly and the basisphenoid medially. A slit between the petrosa and sphenoid appears to be narrower in larger specimens. All scale bars: $1 \mathrm{~mm}$. chiasma, optic chiasma; HP, hypophysis; ICA, internal carotid artery; ON, optic nerve; SPS, superior petrosal sinus; TG, trigeminal ganglion. 
term. The ICA passed through a wide gap or loose mesenchymal space between the cochlear wall and sphenoid. The anteroposterior length of the gap was almost same as the short diameter of the cochlear turn or spiral.

In the anterior side of the longitudinal course of the ICA, a single sagittal section was likely to contain three elements of the sphenoid: the ala temporalis, the alar process and the pterygoid (Fig. 2E). The pterygoid was always ossified except for the hamulus. However, whether the ala temporalis and alar process were cartilaginous or bony varied between specimens even in specimens of almost same sizes. Thus, three combinations were seen: 1) the ala and alar process were bony (Fig. 2B), 2) the alar process was bony and the ala temporalis was cartilaginous (Fig. 2D), 3) both were cartilaginous (Fig. 2E). The third pattern was seen in specimens of CRL $75 \mathrm{~mm}, 80 \mathrm{~mm}$, and $105 \mathrm{~mm}$. The cartilaginous ala looked like the pterygoid in shape (Fig. 2D). The pterygoid contained two types of ossification: 1) endochondral ossification that was characterized by balloon-like cartilage cells adjacent to eosinophilic calcified zone and 2) membranous ossification without cartilage cells at and near the calcified zone (Fig. 2F). Posterior parts of the pterygoid, those faced the ICA, took endochondral ossification.

Although it did not depend on sizes of fetuses, topographical anatomy of the pharyngotympanic tube (PTT) varied between specimens: the PTT was closely to or distant from the ala temporalis and ICA (Fig. 2B vs. Fig. 2D, E). Notably, a linear mesenchymal condensation, crossing the ICA course, extended between the PTT cartilage and the cochlear wall (Fig. 2B, D, E). Being different from the adult morphology, a posterolateral part of the trigeminal ganglion was attached to the cochlear wall (Fig. 2B, C).

According to horizontal sections from five midterm fetuses (Fig. 3), the ICA was surrounded by the petrosa or cochlear wall posteriorly, the alar process anteriorly and the basisphenoid (body of the sphenoid) medially. The basisphenoid is a large cartilaginous mass that contains both of the hypophysial fossa (Rathke's pouch remnant) and the notochord rostral end $[15,16]$. The basisphenoid connected to the petrosal cochlear wall. A slit between the alar process and patrosa became narrower in larger specimens (Fig. 3D vs. Fig. 3F). Likewise, the ICA course was also constricted by another slit of the sphenoid between the basisphenoid and the orbital cartilage (future ala minor) (Fig. 3A). As shown in sagittal sections, the trigeminal ganglion was attached to the cochlear wall (Fig. 3A). Taken together with observations of sagittal sections, at midterm, the ICA did not face areas of membranous ossification but it was completely surrounded by endochondral ossification sites.

\section{Observations of the late-stage fetuses}

We had an impression that the ICA consistently passed through a narrow gap between the bony alar process and the bony cochlear wall for entering a parasellar area of the future cavernous sinus (Figs. 4-6). However, this "narrow gap" had the almost same anteroposterior length as the short diameter of the cochlear turn or spiral. Thus, the gap appeared not to be narrower than that at midterm. The alar process and ala temporalis of the sphenoid became large in combination with the thickening cochlear wall. In contrast to the bony alar process, the ala temporalis still contained a large cartilage mass in the center (Figs. 4D, 5A, 6A), whereas the pterygoid was composed of multiple bony bars (Fig. 5A). Below the gap between the sphenoid and cochlear wall, the ICA was contained in a dense mesenchymal tissue of which inferior margin was connected between the sphenoid (the alar process, ala temporalis and/or pterygoid) and the anterior cochlear wall (Figs. 5A, B, 6D). In addition, as in midterm fetuses, the trigeminal ganglion was always attached to the cochlear wall (Figs. 4B, C, 5D, 6F).

Irrespective of the fetus sizes, below the alar process of the sphenoid, a structure anterior to the ICA course was different between specimens and it was classified into three morphologies: 1) the dense mesenchymal tissue contained abundant veins (Fig. 4; CRL, $228 \mathrm{~mm}$ ); 2) membranous ossification occurred in the dense mesenchymal tissue (Fig. 5; CRL, $276 \mathrm{~mm}$ ) and; 3) a bony process became surrounding the ICA in contrast to the highly reduced size of dense mesenchymal tissue (Fig. 6; CRL, $274 \mathrm{~mm}$ ). The dense mesenchymal tissue with ossification spots was connected with the cochlear wall rather than the sphenoid because the PTT interposed between the dense tissue and the sphenoid (Fig. $5 \mathrm{D})$. The bony process surrounding the ICA was not originated from the sphenoid but the cochlear wall (Fig. 6B, E) and it continued to an anteromedial end of the tympanic cavity wall (Fig. 6F). Thus, conversely, the ICA became positioned in the supero-antero-lateral side of the tympanic cavity (Fig. 7A, B). A fatty tissue occupied a large loose space below the dense mesenchymal tissue (Fig. 5A), i.e., the prestyloid compartment of the parapharyngeal space $[17,18]$ and it also contained muscles from the stylopharyngeus and levator veli palatini muscles. 


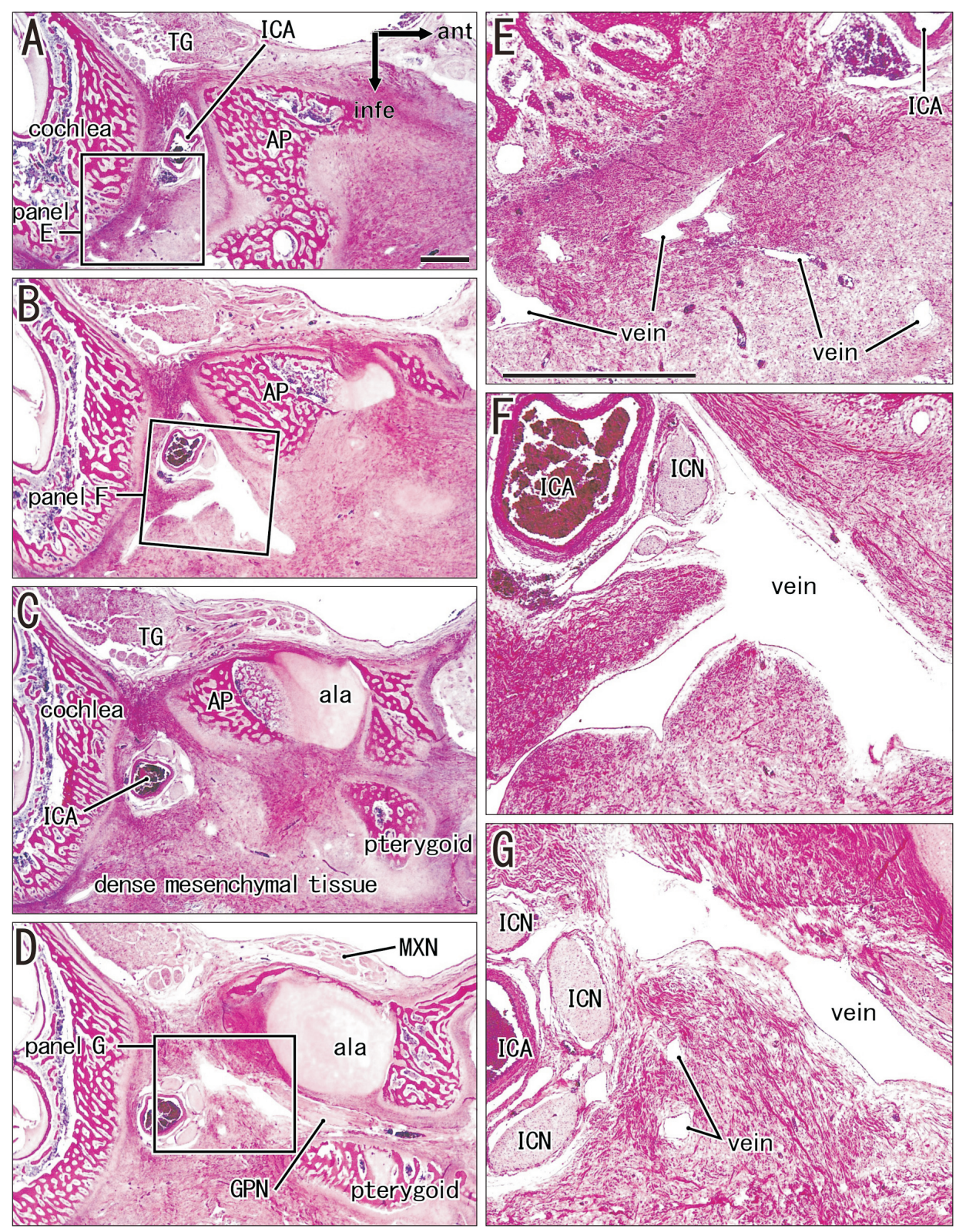

Fig. 4. Wide and dense mesenchymal tissue containing the internal carotid artery and extending between the cochlear wall and sphenoid. Horizontal sections. A fetus of crown-rump length (CRL) $228 \mathrm{~mm}$ (approximately 28 weeks). H\&E staining. (A) or (D) displays the most superior (or inferior) site in the figure. (E-G) are higher magnification views of squares in (A), (B), and (D), respectively. The left-hand side of each panel corresponds to the anterior side of the head. In these superior sections, no structure delineates the future carotid canal except for the bony alar process (AP) of the sphenoid. Instead, a dense mesenchymal tissue extends between the cochlear wall and sphenoid and it contains abundant veins. (A-D) or (E-G) were prepared at the same magnification; (A, E) Scale bar: $1 \mathrm{~mm}$. ala, ala temporalis of the sphenoid; GPN, greater petrosal nerve; ICA, internal carotid artery; ICN, internal carotid nerve; MXN, maxillary nerve (intracranial course); TG, trigeminal ganglion.

Even after the anterior cochlear wall involved the ICA inside (the aforementioned, the third morphology), a septum was underdeveloped between the tympanic cavity and the future carotid canal: membranous ossification was on-going in the septum (Figs. 6F, 7D, E). Likewise, a separation of the facial nerve from the tympanic cavity by a membranous bone was always incomplete (Fig. 7C), i.e., the so-called facial canal dehiscence (see the Introduction). In contrast to these membranous bones, a tidemark (a border between the calcified and uncalcified bones) was sometimes seen in bones near 


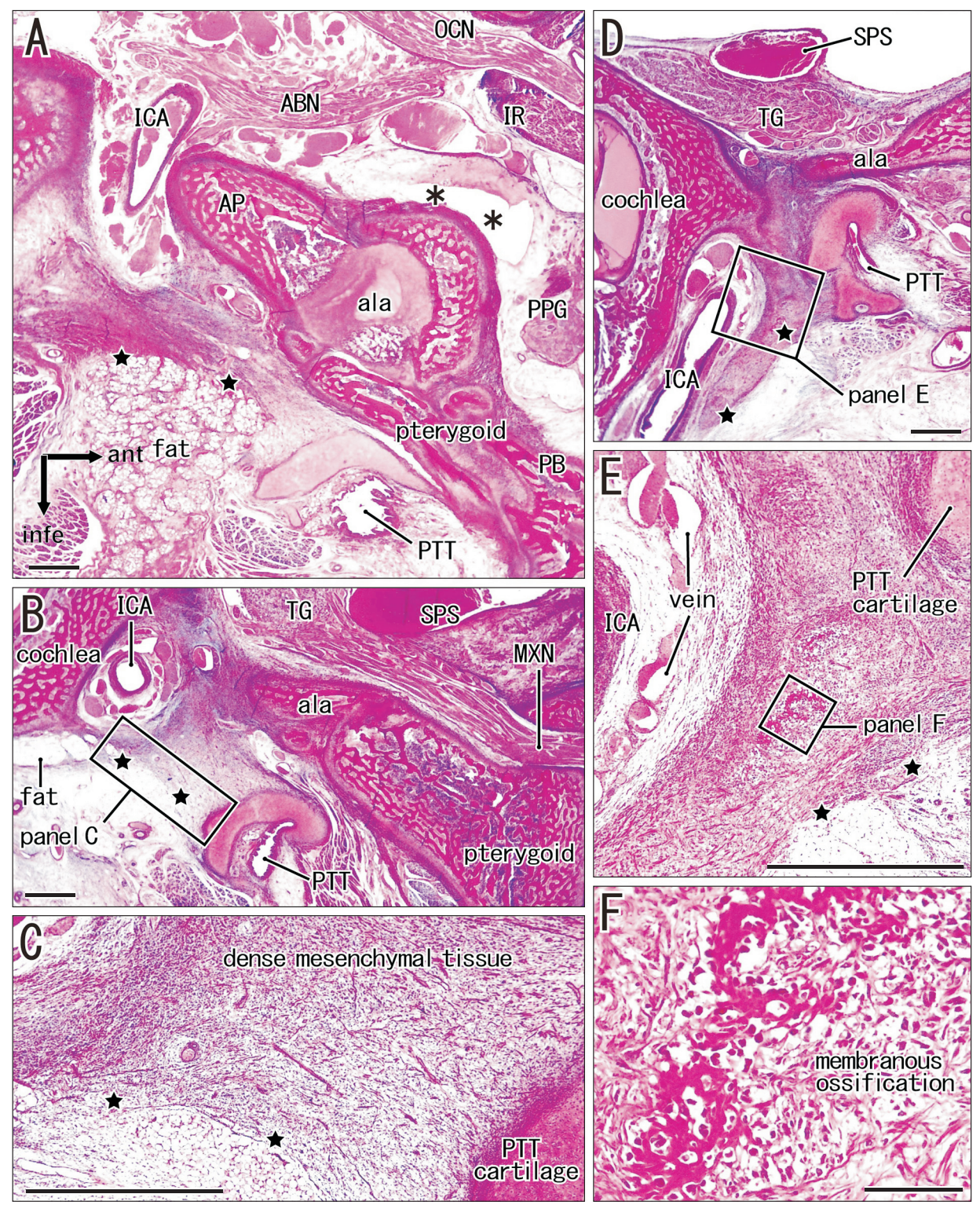

Fig. 5. Initial ossification of the carotid canal starts in the dense mesenchymal tissue connecting to the anterosuperior end of the cochlear wall. Sagittal sections. A fetus of crown-rump length (CRL) $276 \mathrm{~mm}$ (approximately 32 weeks). H\&E staining. Panel (A) containing the future cavernous sinus, displays the most medial site in the figure. (C) and (E) are higher magnification views of squares in (B) and (D), respectively. Panel (F) corresponding to a square in (E), exhibits the membranous ossification starts in the in the dense mesenchymal tissue connecting to the anterior end of the cochlear wall. The left-hand side of each panel corresponds to the anterior side of the head. Note an inferior margin (stars in A-E) of the dense mesenchymal tissue extends between the cochlear wall and the sphenoid (the alar process [AP], ala temporalis [ala] and pterygoid): it connects with the pharyngotympanic tube cartilage in (B) and (D). The palatine bone (PB) looks like a part of the pterygoid (A). Scale bars: (A-E) $1 \mathrm{~mm}$; (F) $0.1 \mathrm{~mm}$. ala, ala temporalis of the sphenoid; ABN, abducant nerve; ICA, internal carotid artery; IR, inferior rectus of the eye; MXN, maxillary nerve in the round foramen; OCN, oculomotor nerve; PPG, pterygopalatine ganglion; PTT, pharyngotympanic tube; SPS, superior petrosal sinus; TG, trigeminal ganglion.

and along the membranous labyrinth (two inserts in Fig. 7C).

\section{Discussion}

The present study demonstrated that membranous os- sification occurred in a dense mesenchymal tissue along the ICA. This mother tissue of the bony carotid canal, containing abundant veins, was delineated anteriorly and posteriorly by the cartilaginous petrosa and sphenoid, the latter was composed of the basisphenoid medially, the alar process 

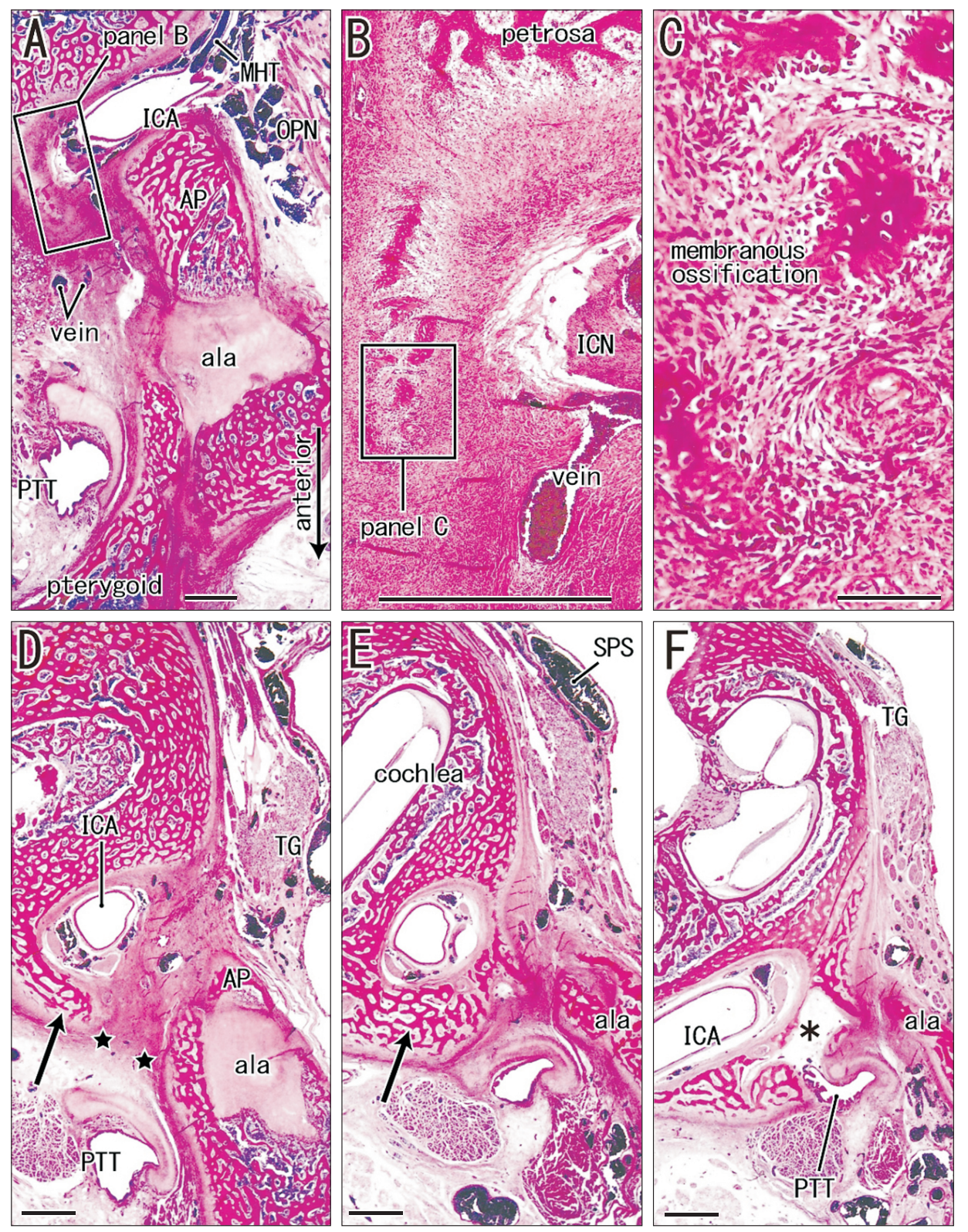

Fig. 6. Carotid canal is involved into the bony cochlear wall. Sagittal sections. A fetus of crown-rump length (CRL) 274 mm (approximately 31 weeks). H\&E staining. The left-hand side of each panel corresponds to the superior side of the head. Panel (A) containing the future cavernous sinus, displays the most medial site in the figure. (B) or (C) is a higher magnification view of a square in (A) or (B). The inferior side of each panel corresponds to the anterior side of the head (long arrow in A). In the medial part of the carotid canal (A-C), a membranous ossification is ongoing. In contrast, in the lateral part, a bony process of the cochlear wall (arrow in D, E) appears to surround the carotid canal from the inferior side rather than from the superior side (Fig. 3D). Stars in (D) indicate an inferior margin of the dense mesenchymal tissue (corresponding to that in Fig. 3A, B) extends between the cochlear wall and sphenoid (ala temporalis or ala). Asterisk in (F) indicates the medial end of the growing tympanic cavity. Scale bars: (A, B, D-F) $1 \mathrm{~mm}$; (C) $0.1 \mathrm{~mm}$. AP, alar process of the sphenoid; ICA, internal carotid artery; ICN, internal carotid nerve; MHT, meningohypophysial trunk of the internal carotid artery; OPN, ophthalmic nerve; PTT, pharyngotympanic tube; SPS, superior petrosal sinus; TG, trigeminal ganglion.

supero-anteriorly, the ala temporalis infero-anteriorly. The pterygoid of the sphenoid also makes an infero-anterior margin. Notably, all these sphenoid elements facing the ICA took endochondral ossification. Thus, any sites of membra- nous ossification did not face the mother tissue of the bony carotid canal. Therefore, at the initial stage, membranous ossification along the ICA seemed to be likely in both or either mesenchymal tissues derived from the petrosa and sphenoid. 

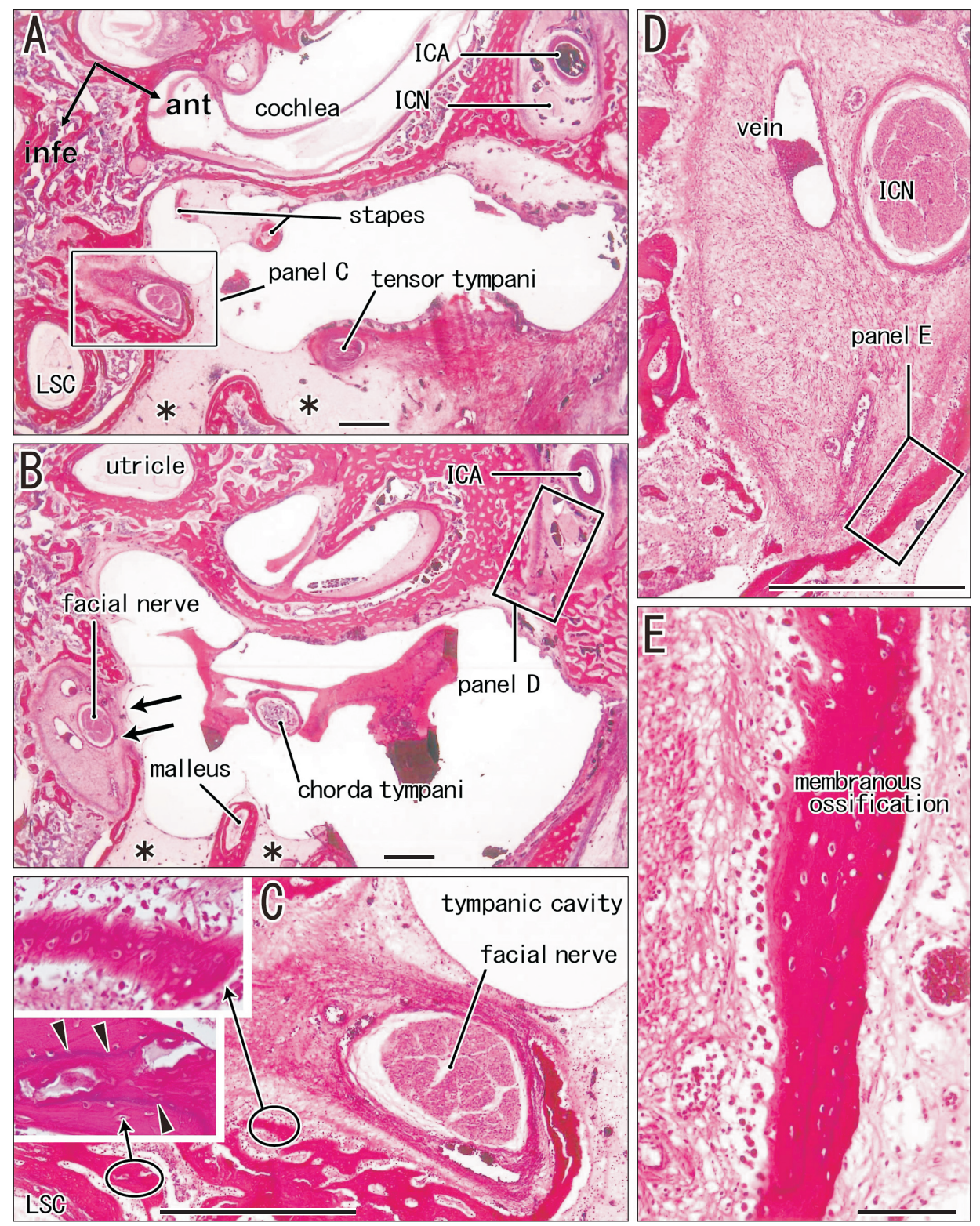

Fig. 7. Carotid and facial nerve canals adjacent to the tympanic cavity: membranous ossification in these septa. Sagittal sections. A fetus of crown-rump length (CRL) $272 \mathrm{~mm}$ (approximately 31 weeks). H\&E staining. The left-hand side of each panel corresponds to the posterior side of the head. Panel (A) displays a plane $1.2 \mathrm{~mm}$ medial to (B). (A, B) Contain the carotid and facial nerve canals: the septum is developing for the internal carotid artery (ICA), while the latter canal still opens widely to the tympanic cavity (arrows in B). (C) or (E) is a higher magnification view of a square in panel (A) or (D). The left-hand side of each panel corresponds to the anterior side of the head. Near the facial nerve, membranous ossification (upper insert of C) coexist endochondral ossification indicated by a tidemark (arrowheads; lower insert). (D) A section near (B), exhibits a site corresponding to a square in (B). (E) A higher magnification view of a square in (D), shows membranous ossification in a septum between the carotid canal and tympanic cavity. Scale bars: (A-D) $1 \mathrm{~mm}$; (E) $0.1 \mathrm{~mm}$. Magnification of inserts, same as (E). ant, anterior; infe, inferior; ICN, internal carotid nerve; LSC, lateral semicircular canal.

The ICA comes from the parapharyngeal space far posterior to the sphenoid $[17,18]$. The ICA seemed to be pulled posteriorly at the cranial base: this traction might be one reason of no or little contribution of a mesenchymal tissue from the sphenoid for the carotid canal ossification.
A narrow gap between the alar process and cochlear wall early determined the superior end of the future bony carotid canal. However, not the alar process but the basisphenoid contributes to a connection between the petrosa and sphenoid. Being different from an imagination suggested by the 
name, the ala temporalis of the sphenoid does not connect with the petrosa but builds a part of the skull base for a base of the inferiorly extending pterygoid. Inferiorly, the mother tissue of the bony canal was delineated by the PTT cartilage at midterm. The PTT cartilage appears late and finally in all cartilages of the body [1]. Near term, the growing pterygoid pushed the PTT upward and, in turn, the PTT pushed the ICA upward toward the petrosa. The pterygoid enlarged to provide large attachments of pterygoid muscles. Simultaneously, the PTT separated the mother tissue of the bony canal from the pterygoid. Therefore, the newly-built membranous bone along the ICA was liable to attach to the petrosa, not to the sphenoid. Depending on involvement of the PTT into the bony tympanic cavity (or depending on growth of the tympanic bone), the ICA was also involved into the petrosa. The bony carotid canal developed without a clear correlation with size of fetuses after 25 weeks. The final process, ossification of the septum between the carotid canal and tympanic cavity, was much delayed and possibly finished after birth.

At any stage examined, the trigeminal ganglion attached to the cochleal wall in contrast to the adult morphology and the ICA did not show the siphon-like course alongside the sphenoid. The former morphology suggested a drastic topographical change or an anterior sliding of the ganglion after birth. However, magnetic resonance imaging of fetuses and infants did not state about the change [19-22]. The latter evoked a question as to when and how multiple turns or a siphon-like course of the ICA is established: 1) an anteromedial turn at the base of the petrosa (an external aperture of the carotid canal);2) an anterior bending at the entrance to the cavernous sinus; 3 ) a strong posterior bending in the sinus; 4 ) a superior turn at the exit from the cavernous sinus. However, Weninger and Müller [23, 24] demonstrated that, in infants, the intracavernous ICA shows an individual and age-dependent variations from the almost straight course (25\%) to an adult-like siphon. Our group recently ensured the straight course in near-term fetuses [25].

\section{ORCID}

Yohei Honkura: https:/orcid.org/0000-0003-1618-3908

Masahito Yamamoto:

https:/orcid.org/0000-0001-9683-6678

José Francisco Rodríguez-Vázquez:

https://orcid.org/0000-0001-5423-4492

Gen Murakam: https://orcid.org/0000-0001-7181-5475
Hiroshi Abe: https://orcid.org/0000-0002-4683-4487

Shin-ichi Abe: https://orcid.org/0000-0003-4632-9736

Yukio Katori: https://orcid.org/0000-0003-1963-5551

\section{Author Contributions}

Conceptualization: GM. Data acquisition: YH, MY. Data analysis or interpretation: JFRV. Drafting of the manuscript: HA, SA. Critical revision of the manuscript: YK. Approval of the final version of the manuscript: all authors.

\section{Conflicts of Interest}

No potential conflict of interest relevant to this article was reported.

\section{Acknowledgements}

This study was supported by Grant-in Aids for Scientific Research (No.18H02949 for Yukio Katori, No.18K16826 for Yohei Honkura and No.16K08435 for Hiroshi Abe) from the Ministry of Education, Culture, Sports, Science and Technology, Japan.

\section{References}

1. Katori Y, Rodríguez-Vázquez JF, Verdugo-López S, Murakami G, Kawase T, Kobayashi T. Initial stage of fetal development of the pharyngotympanic tube cartilage with special reference to muscle attachments to the tube. Anat Cell Biol 2012;45:185-92.

2. Jin ZW, Cho KH, Abe H, Katori Y, Murakami G, RodríguezVázquez JF. Fetal facial nerve course in the ear region revisited. Surg Radiol Anat 2017;39:885-95.

3. Zhang Q, Wang H, Udagawa J, Otani H. Morphological and morphometric study on sphenoid and basioccipital ossification in normal human fetuses. Congenit Anom (Kyoto) 2011;51:13848.

4. Santaolalla-Montoya F, Martinez-Ibargüen A, Sánchez-Fernández JM, Sánchez-del-Rey A. Principles of cranial base ossification in humans and rats. Acta Otolaryngol 2012;132:349-54.

5. Hayashi S, Kim JH, Hwang SE, Shibata S, Fujimiya M, Murakami $\mathrm{G}$, Cho $\mathrm{BH}$. Interface between intramembranous and endochondral ossification in human foetuses. Folia Morphol (Warsz) 2014;73:199-205.

6. Michaels L, Soucek S, Linthicum F. The intravestibular source of the vestibular aqueduct. II: its structure and function clarified by a developmental study of the intra-skeletal channels of the otic capsule. Acta Otolaryngol 2010;130:420-8.

7. Bast TH, Anson BJ. The temporal bone and the ear. Springfield: 
Thomas; 1949.

8. Anson BJ, Donaldson JA. Surgical anatomy of the temporal bone and ear. 2nd ed. Philadelphia: WB Saunders; 1973.

9. Nemzek WR, Brodie HA, Chong BW, Babcook CJ, Hecht ST, Salamat S, Ellis WG, Seibert JA. Imaging findings of the developing temporal bone in fetal specimens. AJNR Am J Neuroradiol 1996;17:1467-77.

10. Richard C, Courbon G, Laroche N, Prades JM, Vico L, Malaval L. Inner ear ossification and mineralization kinetics in human embryonic development- microtomographic and histomorphological study. Sci Rep 2017;7:4825.

11. Abing W, Rauchfuss A. Fetal development of the tympanic part of the facial canal. Arch Otorhinolaryngol 1987;243:374-7.

12. Spector JG, Ge X. Ossification patterns of the tympanic facial canal in the human fetus and neonate. Laryngoscope 1993;103:1052-65.

13. Yamamoto M, Kitamura K, Kasahara M, Serikawa M, Katumura S, Yoshimoto T, Matubayashi T, Odaka K, Matsunaga S, Abe S. Histological study of the developing pterygoid process of the fetal mouse sphenoid. Anat Sci Int 2017;92:364-72.

14. Yamamoto M, Takada H, Ishizuka S, Kitamura K, Jeong J, Sato M, Hinata N, Abe S. Morphological association between the muscles and bones in the craniofacial region. PLoS One 2020;15:e0227301.

15. Katori Y, Kawamoto A, Cho KH, Ishii K, Abe H, Abe S, Rodríguez-Vázquez JF, Murakami G, Kawase T. Transsphenoidal meningocele: an anatomical study using human fetuses including report of a case. Eur Arch Otorhinolaryngol 2013;270:272936.

16. Cho KH, Chang H, Yamamoto M, Abe H, Rodríguez-Vázquez JF, Murakami G, Katori Y. Rathke's pouch remnant and its regression process in the prenatal period. Childs Nerv Syst 2013;29:761-9.
17. Katori Y, Kawase T, Cho KH, Abe H, Rodríguez-Vázquez JF, Murakami G, Abe S. Prestyloid compartment of the parapharyngeal space: a histological study using late-stage human fetuses. Surg Radiol Anat 2012;34:909-20.

18. Katori Y, Kawase T, Ho Cho K, Abe H, Rodríguez-Vázquez JF, Murakami G, Fujimiya M. Suprahyoid neck fascial configuration, especially in the posterior compartment of the parapharyngeal space: a histological study using late-stage human fetuses. Clin Anat 2013;26:204-12.

19. Sánchez-Fernández JM, Saint-Gerons S, Sánchez del Rey A. A microanalytical study on human auditory ossicles in normal and pathological conditions. Acta Otolaryngol 1992;112:317-21.

20. Ohtsuki S, Ishikawa A, Yamada S, Imai H, Matsuda T, Takakuwa T. Morphogenesis of the middle ear during fetal development as observed via magnetic resonance imaging. Anat Rec (Hoboken) 2018;301:757-64.

21. Nemzek WR, Brodie HA, Hecht ST, Chong BW, Babcook CJ, Seibert JA. MR, CT, and plain film imaging of the developing skull base in fetal specimens. AJNR Am J Neuroradiol 2000;21:1699-706.

22. Jeffery N, Spoor F. Ossification and midline shape changes of the human fetal cranial base. Am J Phys Anthropol 2004;123:78-90.

23. Weninger WJ, Müller GB. The sympathetic nerves of the parasellar region: pathways to the orbit and the brain. Acta Anat (Basel) 1997;160:254-60.

24. Weninger WJ, Müller GB. The parasellar region of human infants: cavernous sinus topography and surgical approaches. J Neurosurg 1999;90:484-90.

25. Sato M, Cho KH, Yamamoto M, Hirouchi H, Murakami G, Abe H, Abe S. Cavernous sinus and abducens nerve in human fetuses near term. Surg Radiol Anat 2020;42:761-70. 